Reprod. Nutr. Dévelop., 1988, 28 (1), 179-180.

\title{
Sécrétions pancréatico-biliaires et profil moteur de l'intestin grêle chez le mouton
}

\author{
C. H. MALBERT, Y. RUCKEBUSCH
}

Laboratoire de Physiologie, Ecole Nationale Vétérinaire, 23, chemin des Capelles, 31076 Toulouse Cedex, France.

Summary. The influence of biliopancreatic secretions (BPS) on duodenal motility was assessed in conscious sheep fitted with electrodes before and beyond the bile duct. The regular spiking activity phase of the migrating motor complexes (MMC) faded out and the MMC intervals became irregular within $24 \mathrm{~h}$ beyond the bile duct after BPS withdrawal. The normal MMC pattern was restored by intraluminal infusion of BPS or sodium hypochlorite probably by counteracting bacterial overgrowth.

Chez le mouton, les sécrétions pancréatico-bilaires SPB ne sont mélangées au chyme gastrique que $20 \mathrm{~cm}$ en aval du pylore, d'où un $\mathrm{pH}$ du contenu de la partie proximale du duodénum voisin de celui de la caillette (Malbert et Ruckebusch, 1987). Le caractère cyclique de la motricité à ce niveau n'est pas différent de celui trouvé sur la partie distale du duodénum en aval de l'abouchement du canal cholédoque. Or, des perturbations motrices de l'intestin sont souvent associées à la rétention biliaire chez les ruminants. Cela nous a conduit à vérifier l'influence possible des SPB sur les caractéristiques du profil moteur du duodénum distal, soit en supprimant l'apport des SPB, soit en assurant l'apport des SPB en zone jéjunale.

Matériel et méthodes. Une dérivation extracorporelle permanente des SPB est assurée au moyen d'un cathéter de silastic fixé dans le canal cholédoque à la hauteur de son abouchement dans la lumière duodénale. Les SPB peuvent être ainsi mesurées et réintroduites en zone distale par un autre cathéter fixé dans la lumière jéjunale pendant 48 à $72 \mathrm{~h}$. Un autre cathéter fixé dans la lumière du duodénum proximal sert à amener les SPB au contact du chyme gastrique $10 \mathrm{~cm}$ en aval du pylore. L'activité électrique gastro-intestinale a été appréciée à partir d'électrodes laissées à demeure environ $10 \mathrm{~cm}$ et $90 \mathrm{~cm}$ en aval du pylore chez 4 brebis adultes de $35-40 \mathrm{~kg}$.

Résultats et discussion. La dérivation externe des SPB associée à leur réintroduction continue $10 \mathrm{~cm}$ en aval du pylore, ne modifie pas la motricité duodénale, en particulier l'initiation périodique de cycles moteurs caractérisés par une phase d'activité régulière (AR) toutes les 90 min environ. En revanche, la simple dérivation externe des SPB ou leur réintroduction en zone jéjunale, s'accompagne dans les heures qui suivent d'une altération motrice de la partie distale du duodénum avec irrégularité de l'intervalle séparant les phases d'AR et réduction de moitié de leur durée (fig. 1). L'irrégularité des complexes myoélectriques migrants en zone distale est maximale de $8 \mathrm{~h}$ à $24 \mathrm{~h}$ après exclusion des SPB sans modification de la motricité en zone proximale ni de l'ingestion alimentaire malgré l'apparition d'un clocher hyperthermique. La restitution de bile (environ $250 \mathrm{ml}$ ) dans la lumière du duodénum proximal restaure en $90 \pm 35 \mathrm{~min}$ le profil moteur duodénal (fig. 1). Pendant les $48 \mathrm{~h}$ de dérivation ou d'exclusion des SPB, la température corporelle s'élève d'environ $1^{\circ} \mathrm{C}$, sans effet correcteur net d'une antibiothérapie par voie générale. En revanche, la perfusion lente de $50 \mathrm{ml}$ d'hypochlorate de sodium en zone duodénale proximale restaure le profil moteur altéré par l'exclusion biliaire en 3 à $4 \mathrm{~h}$ et supprime l'hyperthermie. 
L'analogie entre les effets locaux d'un antiseptique et ceux des SPB suggère l'existence d'une relation entre la désorganisation du profil moteur de l'intestin et une croissance bactérienne accrue telle qu'elle a été observée en clinique humaine (Vantrappen et al., 1977). Expérimentalement, une croissance bactérienne accrue résulterait, chez le rat, de I'hinhibition des phases d'AR (Scott et Cahall, 1982). Nos résultats indiquent que l'absence des SPB au niveau duodénal altère le profil moteur de l'intestin grêle en aval, à l'instar d'une croissance bactérienne incontrôlée. Le fait que cette altération motrice soit supprimée par une substance antiseptique suggère un rôle bactériostatique des sécrétions pancréatico-biliaires chez I'herbivore ruminant.
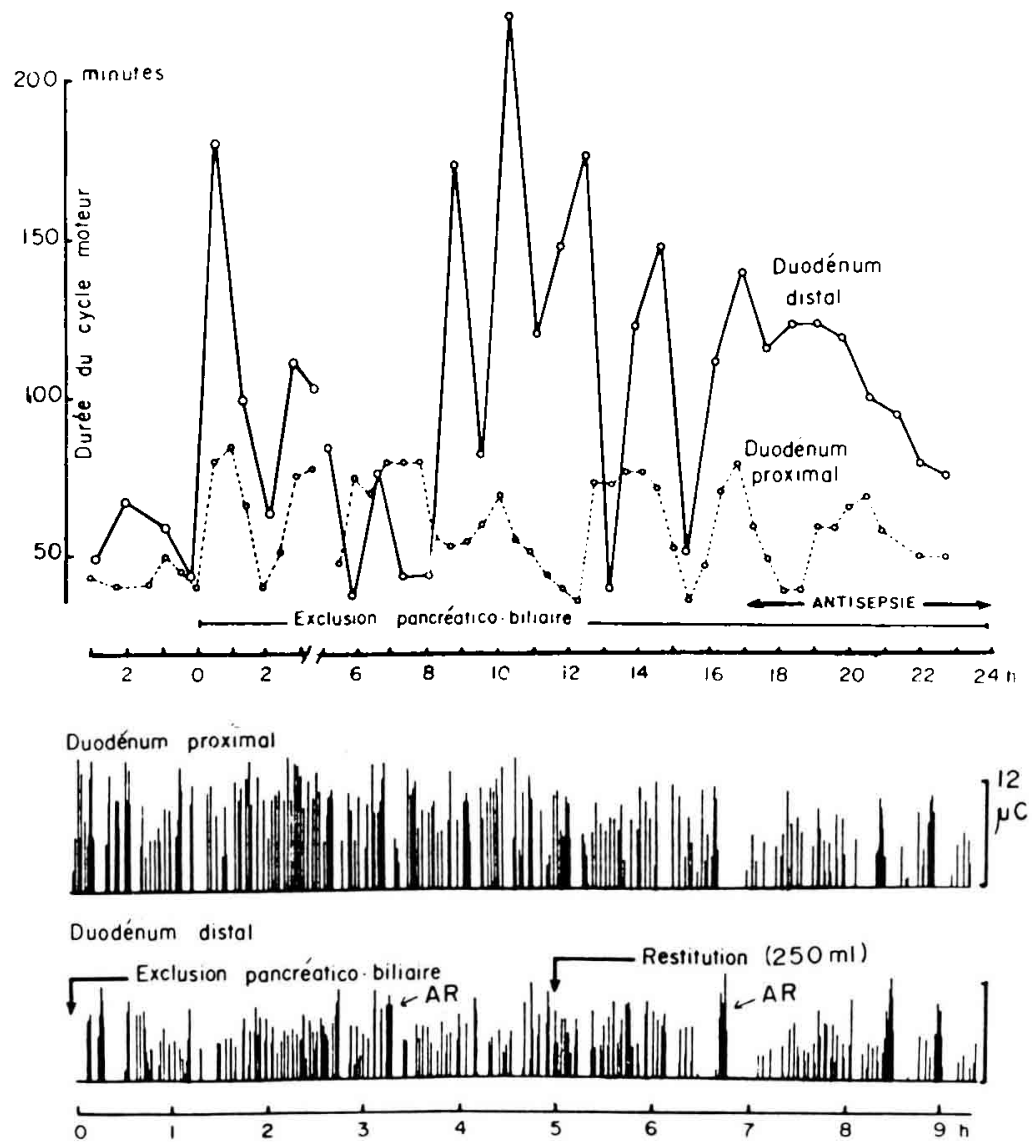

FIG. 1. - Exemple d'augmentation de la durée du cycle moteur de l'intestin au niveau du duodénum distal (en haut) et de la diminution de la durée des phases d'activité régulière (en bas) à la suite de l'exclusion des sécrétions pancréatico-biliaires. La perfusion en une heure de $50 \mathrm{ml}$ d'eau de Javel ramène au bout de $3 \mathrm{~h}$ la durée du cycle moteur à sa valeur normale (en haut); celle de $250 \mathrm{ml}$ de bile restaure le profil moteur du duodénum distal (en bas).

Malbert C. H., Ruckebusch Y., 1987. J. Vet. Med. A, 34, 428-438.

Scott L. D., Cahall D. L., 1982. Gastroenterology, 82, 737-745.

Vantrappen G., Janssens J., Hellemans J., Ghoos Y., 1977. J. Clin. Invest., 59, 1158-1166. 